\title{
Facteurs de variation des paramètres protéo-énergétiques, enzymatiques $B$. Faye' et minéraux dans le plasma C. Mulato - chez le dromadaire de Djibouti
}

FAYE (B.), MULATO (C.). Facteurs de variation des paramètres protéoénergétiques, enzymatiques et minéraux dans le plasma chez le dromadaire de Djibouti. Revue Élev. Méd. vét. Pays trop., 1991, 44 (3) : 325-334

L'analyse du plasma de 52 dromadaires ( 27 femelles adultes, 13 jeunes femelles, 7 mâles adultes et 5 jeunes mâles) en provenance de cinq régions naturelles de Djihouti a concerné 15 paramètres biochimiques. Les valeurs observées tendent à admettre des états de subcarence en oligo-éléments (cuivre : $60,7 \mu \mathrm{g} / 100 \mathrm{ml}$; zinc : $46,2 \mu \mathrm{g} / 100 \mathrm{ml})$ mais non en calcium $(9,5 \mathrm{mg} / 100 \mathrm{ml})$, ni en magnésium $(2,3 \mathrm{mg} / 100 \mathrm{ml})$, bien que dans ce dernier cas les écarts soient grands $(1,1-3,9)$. Par ailleurs, les dromadaires de Djibouti semblent en état de déficit énergétique $(63,7 \mathrm{mg} / 100 \mathrm{ml}$ pour le glucose, $0,025 \mathrm{mmol} / \mathrm{l}$ pour le $\mathbf{B O H}, 0,17 \mathrm{mmol} / \mathrm{l}$ pour les acides gras libres), mais le niveau azoté alimentaire serait globalement satisfaisant (urée : $\mathbf{3 5 , 9} \mathrm{mg} / 100 \mathrm{ml}$, albumine : 32,7 $\mathrm{g} / \mathrm{l}$ ). Les lipides plasmatiques (cholestérol, phospholipides, triglycérides) signent les particularités métaboliques du dromadaire (respectivement 19,5, 22,4 et $26,6 \mathrm{mg} / 100 \mathrm{ml}$ ). Le facteur géographique est prépondérant sur les facteurs zootechniques pour expliquer les différences entre les profils observés. Les caractéristiques des cinq régions sont discutées en relation avec le profil moyen observé sur les animaux. Mots clés : Dromadaire - Biochimie - Enzyme - Teneur en éléments minéraux Plasma - Djibouti.

\section{INTRODUCTION}

Les paramètres plasmatiques peuvent constituer des indicateurs, plus ou moins fidèles, de l'état nutritionnel des animaux. Ils permettent de détecter d'éventuelles carences alimentaires et de diagnostiquer, par ailleurs, des troubles pathologiques subcliniques. Les facteurs de variation physiologiques, voire pathologiques, sont assez bien connus chez la plupart des espèces $(40,44)$. Pour le dromadaire, les données sont plus fragmentaires, voire rarissimes pour certains paramètres, et on ne dispose pas toujours de normes sanguines bien définies.

Le statut nutritionnel des dromadaires, dans le contexte géo-climatique et alimentaire de la République de Djibouti, est essentiellement tributaire des ressources fourragères du milieu. Dans un précédent article (25), ont été examinées les teneurs en oligo-éléments dans les fourrages et le plasma des quatre espèces de ruminants domestiques que l'on rencontre à Djibouti. La présente étude a pour but de déterminer les valeurs de certains paramètres protéo-énergétiques, minéraux et enzyma-

1. Laboratoire d'Écopathologie, INRA Theix, 62122 Ceyrat, France. 2. Mission française de coopération, BP 203, Nouakchott, Mauritanie.

Reçu le 2.2.1990, accepté le 28.5.1991. tiques, en relation avec l'origine géographique des troupeaux (régions naturelles et/ou système de production particulier) et des caractéristiques zootechniques comme l'âge et le sexe.

\section{MATÉRIEL, MÉTHODE, CHOIX DES SITES}

\section{Les animaux et leur environnement}

L'étude porte sur 52 dromadaires de races locales (Dankali et race dite "Grand de Somalie") répartis en 10 mâles et 42 femelles et en 18 jeunes et 34 adultes (animaux en âge de se reproduire). Les animaux proviennent de cinq régions naturelles (carte '1) telles qu'elles sont décrites par AUDRU et al. (7) et FAYE (22).

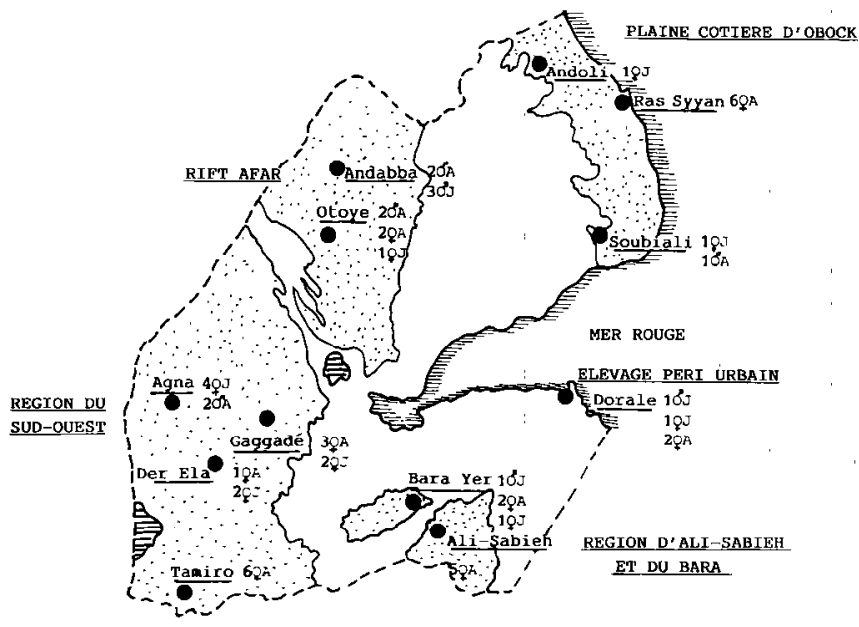

Carte 1 : Régions naturelles étudiées et lieux de prélèvement sanguin des dromadaires mâles ( $ठ)$. femelles $(q)$, adultes $(A)$ oul jeunes $(J)$.

\section{Rift de l'Afar}

Les animaux prélevés pâturent dans la zone nord, caractérisée par une végétation dispersée à Rhighozum somalense entrecoupée par la steppe arbustive d'Andabba à Acacia ehrenbergiana et la zone dénudée d'Otoye. 


\section{Plaine côtière d'Obock}

La plupart des animaux étudiés subsistent dans les mangroves, formations végétales à Ceriops tagal et Avicennia marina. A l'intérieur des terres, on rencontre des fourrés unispécifiques à Salvadora persica.

\section{Région d'Ali-Sabieh}

Cette zone comprend le Grand et le Petit Bara au nord, où cohabitent zones dénudées et steppe à Cymbopogon schoenanthus, et le massif d'Arrey au sud, couvert d'une steppe où dominent Acacia etbaica et des fourrés à Salvadora persica.

\section{Région du Sud-Ouest}

C'est une vaste zone marquée par une série de fossés d'effondrement (Der Elwa, Gaggadé, Hanlé, bordure du lac Abbé) et caractérisée par une steppe arborée à Acacia asak et tortilis, des steppes succulentes à Suaeda, des formations ligneuses à Hyphaene thebaica, des steppes herbeuses à Cymbopogon schoenanthus et des zones inondables à Tamarix niloti$\mathrm{ca}$.

\section{Zones périurbaines}

II ne s'agit pas d'une "région naturelle" mais d'élevages périurbains intensifs (ville de Djibouti), constitués de troupeaux de chamelles laitières destinés à approvisionner les populations urbaines en lait cru. Les animaux reçoivent une alimentation riche en concentrés (sorgho-maïs). Ces zones constituent un système de production particulier.

Pendant la période d'étude, les animaux n'étaient pas en transhumance, donc en situation plutôt stable dans une région donnée.

\section{Prélèvements}

Les prélèvements ont été réalisés au cours du mois de décembre 1988, en saison fraîche et sèche. Les dromadaires ont été soumis à un prélèvement sanguin, de préférence le matin, lorsque les animaux sont concentrés autour des points d'eau. Le sang total est prélevé à la jugulaire dans un tube Sarstedt contenant une goutte d'anticoagulant (Liquemine ${ }^{\mathrm{ND}}$ ), puis centrifugé sur place (4 $500 \mathrm{t} / \mathrm{min}$ pendant $10 \mathrm{~min}$ ) et le plasma collecté avec un filtre à hématies. Le prélèvement plasmatique est conservé à la température de la glace fondante, puis congelé dès le retour à Djibouti-ville, au maximum après un délai de cinq jours à $0^{\circ} \mathrm{C}$.

\section{Analyses de laboratoire et choix des paramètres}

Le statut minéral des animaux est recherché par dosage des macro-éléments (calcium, magnésium) et des oligoéléments (cuivre, zinc). Ces paramètres sont en partie dépendants du statut physiologique de l'animal (cas du calcium en début de lactation) et de l'apport alimentaire. Ces minéraux ont été dosés par spectrophotométrie à absorption atomique, selon la méthode décrite par BELLANGER et LAMAND (11).

Pour les analyses biochimiques et enzymatiques, on a utilisé un analyseur à transfert monocanal informatisé, Isamat (Isa-Biologie), selon les méthodes classiques préconisées pour ce type d'appareil $(8,14,18,19)$. Les paramètres mesurés sont :

- les indicateurs du statut énergétique : glucose, 3hydroxybutyrate $(B O H)$, acides gras libres (AGL);

- les indicateurs du statut azoté : albumine, urée ;

- les indicateurs de l'intégrité organique, en particuliér du foie : gamma glutamyl transférase (GGT) et glutamate déshydrogénase (GLDH) ;

- la céruloplasmine $(C p)$, protéine de transport du cuivre et témoin des processus inflammatoires.

D'autres paramètres de nature lipidique, témoins du statut énergétique des animaux (triglycérides, cholestérol, phospholipides), sont mesurés, respectivement, selon les méthodes de BUCOLO et DAVID (13), ALLAIN et al. (5), et TAKAYAMA et al. (42).

\section{Analyses statistiques}

Les données statistiques classiques (moyenne, écarttype, histogramme) sont établies à partir du logiciel STAT-ITCF (10). Pour la comparaison de populations remplissant les conditions d'homoscédasticité, on a utilisé l'analyse de variance (calcul de F), et dans le cas contraire le test $U$ de MANN et WITHNEY. La comparaison des moyennes observées s'est appuyée sur le test $t$ classique. Les analyses descriptives multidimensionnelles (27) utilisées sont : l'analyse en composantes principales (ACP) et l'analyse discriminante (AD).

\section{RÉSULTATS}

On examinera successivement les valeurs enregistrées sur l'ensemble de l'échantillon, leur variation en fonction de l'origine géographique des animaux et les facteurs discriminants prioritaires (âge, $s \in x \Theta$, région naturelle). 


\section{Valeurs observées sur l'ensemble de l'échantillon (tabl. I)}

\section{Paramètres protéo-énergétiques}

Les glycémies (en $\mathrm{mg} / 100 \mathrm{ml}$ ) sont significativement plus basses chez les femelles $(60,3 \pm 25,3$ vs $78 \pm 7,3)$ par rapport aux mâles et chez les adultes par rapport aux jeunes $(58,5 \pm 25,4$ vs $73,4 \pm 23,7)$. Dix-sept p. 100 des animaux ont des valeurs de $\mathrm{BOH}$ inférieures à la limite de sensibilité des appareils de mesure et on ne note pas de variation significative selon l'âge ou le sexe. L'albumine $(\mathrm{g} / \mathrm{l})$ varie peu, mais on note une différence significative entre les jeunes $(31,3 \pm 3,5)$ et les adultes $(33,5 \pm 3,3)$. La teneur en urée plasmatique $(\mathrm{mg} / 100 \mathrm{ml})$ subit également de grandes variations (rapport de 1 à 10 entre les valeurs extrêmes) et les mâles présentent des valeurs significativement plus faibles $(23,3 \pm 13,5$ vs $39 \pm 17,2)$. Les teneurs en AGL (mmol/l) ont une distribution bimodale et des valeurs plus basses chez les femelles $(0,16 \pm 0,11$ vs $0,23 \pm 0,05)$. Le cholestérol ne présente aucune variation selon l'âge ou le sexe. La teneur en phospholipides ( $\mathrm{mg} / 100 \mathrm{ml})$ ne varie pas non plus en fonction de l'âge, mais on note des valeurs plus faibles chez les femelles $(21,3 \pm 5,9$ vs $27,1 \pm 8,2)$. A l'inverse, les triglycérides ( $\mathrm{mg} / 100 \mathrm{ml}$ ) ne varient pas en fonction du sexe, mais sont inférieurs chez les jeunes $(21,5 \pm 7,6$ vs $36,2 \pm 15,4)$.

\section{Paramètres minéraux}

La dispersion des valeurs de la calcémie $(\mathrm{mg} / 100 \mathrm{ml})$ est étroite et on constate des valeurs plus basses chez les animaux adultes $(10,83 \pm 0,5$ vs $11,3 \pm 0,6)$, mais pas de

TABLEAU I Teneurs plasmatiques observées en éléments minéraux, biochimiques et enzymatiques chez le dromadaire à Djibouti.

\begin{tabular}{|c|c|c|c|c|c|}
\hline $\begin{array}{c}\text { Paramètres } \\
\text { plasmatiques }\end{array}$ & Moyenne & $\begin{array}{c}\text { Valeurs } \\
\text { extrêmes }\end{array}$ & $\begin{array}{l}\text { Écart- } \\
\text { type }\end{array}$ & Unités & $\begin{array}{l}\text { Normes } \\
\text { bovines }\end{array}$ \\
\hline $\begin{array}{l}\text { Cuivre } \\
\text { Zinc } \\
\text { Calcium } \\
\text { Magnésium }\end{array}$ & $\begin{array}{r}60,7 \\
46,2 \\
9,5 \\
2,3\end{array}$ & $\begin{array}{c}7-122 \\
9,2-100 \\
8,5-11,3 \\
1,1-3,9\end{array}$ & $\begin{array}{c}23,4 \\
17,5 \\
0,58 \\
0,62\end{array}$ & $\begin{array}{l}\mu \mathrm{g} / 100 \mathrm{ml} \\
\mu \mathrm{g} / 100 \mathrm{ml} \\
\mathrm{mg} / 100 \mathrm{ml} \\
\mathrm{mg} / 100 \mathrm{ml}\end{array}$ & $\begin{array}{c}70-120 \\
70-120 \\
9-10,5 \\
2-2,8\end{array}$ \\
\hline $\begin{array}{l}\text { Glucose } \\
\beta 0 H \\
\text { Urée } \\
\text { Albumine } \\
\text { Céruloplasmine } \\
\text { AGL } \\
\text { Cholestérol } \\
\text { Phospholipides } \\
\text { Triglycérides }\end{array}$ & $\begin{array}{c}63,7 \\
0,025 \\
35,9 \\
32,7 \\
35,5 \\
0,17 \\
19,5 \\
22,4 \\
26,6\end{array}$ & $\begin{array}{c}12-114 \\
0-0,12 \\
10,8-97,1 \\
21,4-39,1 \\
31,8-41,4 \\
0-0,57 \\
9-52 \\
13-45 \\
9-74\end{array}$ & $\begin{array}{l}25,6 \\
0,026 \\
17,5 \\
3,5 \\
2,9 \\
0,12 \\
8,7 \\
6,7 \\
12,9\end{array}$ & $\begin{array}{c}\mathrm{mg} / 100 \mathrm{ml} \\
\mathrm{mmol} / \mathrm{l} \\
\mathrm{mg} / 100 \mathrm{ml} \\
\mathrm{g} / \mathrm{l} \\
\mathrm{U} 0 \\
\mathrm{mmol} / \mathrm{l} \\
\mathrm{mg} / 100 \mathrm{ml} \\
\mathrm{mg} / 100 \mathrm{ml} \\
\mathrm{mg} / 100 \mathrm{ml}\end{array}$ & \begin{tabular}{|c|}
$55-65$ \\
$0,2-1$ \\
$15-30$ \\
$3-3,8$ \\
$30-55$ \\
$0,2-0,5$ \\
$25-40$ \\
$150-250$ \\
$5-15$
\end{tabular} \\
\hline $\begin{array}{l}\text { GGT } \\
\text { GLDH }\end{array}$ & $\begin{array}{r}8,4 \\
14,1\end{array}$ & $\begin{array}{l}4-20 \\
0-97\end{array}$ & $\begin{array}{r}3,1 \\
21,3\end{array}$ & $\begin{array}{l}\mathrm{UI} / \mathrm{I} \\
\mathrm{UI} / \mathrm{I}\end{array}$ & $\begin{array}{r}12-18 \\
5-11\end{array}$ \\
\hline
\end{tabular}

différences liées au sexe. Dans l'échantillon, on remarque une répartition bimodale des valeurs en magnésium plasmatique. Les taux observés $(\mathrm{mg} / 100 \mathrm{ml})$ chez les femelles sont significativement plus faibles que chez les mâles $(1,1 \pm 0,6$ vs $2,3 \pm 0,2)$, mais on ne relève aucune différence liée à l'âge.

Les valeurs du cuivre et du zinc plasmatique $(\mu \mathrm{g} / 100 \mathrm{ml})$ indiquent une grande dispersion, avec une distribution normale. La cuprémie chez les dromadaires adultes est significativement plus faible que chez les jeunes $(56,4 \pm$ 25,7 vs $68,8 \pm 15,9$ ) et moins élevée chez les femelles que chez les mâles $(55,4 \pm 21,8$ vs $83,1 \pm 16,2)$. On ne note aucune différence pour le zinc.

Le taux de céruloplasmine (unités oxydasiques) est inférieur chez les animaux adultes $(34,4 \pm 2,8$ vs $36,6 \pm 2,8)$ et chez les femelles $(34,7 \pm 2,6$ vs $38,9 \pm 1,2)$ à l'instar de ce qui se passe pour la cuprémie, avec laquelle la céruloplasmine est corrélée.

\section{Paramètres enzymatiques}

Témoins plus ou moins spécifiques des atteintes hépatiques, les paramètres enzymatiques dépassent les seuils - jugés critiques chez les bovins - dans 13,5 p. 100 des cas pour la GLDH. II s'agit dans la plupart des cas de femelles pour lesquelles les valeurs moyennes (en UI/I) sont significativement plus élevées $(16,1 \pm 3,5$ vs $5,4 \pm$ $3,1)$ que chez les mâles. On ne note aucune variation liée à l'âge. La GGT ne varie pas en fonction de l'âge ou du sexe des animaux.

\section{Variations géographiques (tabl. II)}

Les glycémies sont significativement inférieures dans la région du Sud-Ouest et la plaine d'Obock, à l'inverse des animaux en zone périurbaine et du Rift de l'Afar, qui par ailleurs présentent des taux élevés en $B O H$. L'urémie est plus basse dans le Rift de l'Afar et surtout dans la plaine d'Obock. C'est dans cette région qu'on note également les valeurs les plus élevées en AGL.

En revanche, les paramètres du métabolisme lipidique ne varient pas selon les régions, de même que l'albuminémie. La céruloplasminémie, comme la cuprémie, est plus faible dans la région du Sud-Ouest et dans la plaine côtière d'Obock.

La cuprémie est significativement plus basse chez les animaux de la plaine côtière d'Obock ; la zincémie est inférieure chez les femelles laitières des zones périurbaines et de la région d'Ali-Sabieh. Aucune variabilité régionale ne concerne la calcémie, ce qui paraît logique compte tenu des mécanismes de contrôle homéostatique bien connus pour cet élément. En revanche, le taux de magnésium plasmatique est plus faible chez les animaux de la plaine côtière d'Obock et de la région du SudOuest. 
B. Faye C. Mulato

TABLEAU II Moyennes des teneurs plasmatiques des éléments minéraux, biochimiques et enzymatiques chez le dromadaire dans cinq zones géographiques de Djibouti.

\begin{tabular}{|c|c|c|c|c|c|c|}
\hline Paramètres & $\begin{array}{l}\text { Rift de } \\
\text { l'Afar }\end{array}$ & $\begin{array}{c}\text { Plaine } \\
\text { côtière } \\
\text { d'Obock }\end{array}$ & $\begin{array}{c}\text { Élevages } \\
\text { périurbains }\end{array}$ & $\begin{array}{c}\text { Grand Bara } \\
\text { Ali-Sabieh }\end{array}$ & $\begin{array}{l}\text { Sud- } \\
\text { Ouest }\end{array}$ & Unités \\
\hline $\begin{array}{l}\text { Cuivre } \\
\text { Zinc } \\
\text { Calcium } \\
\text { Magnésium }\end{array}$ & $\begin{array}{c}88,9 \\
42,6 \\
9,14 \\
2,65\end{array}$ & $\begin{array}{r}33,67 \\
50,22 \\
9,76 \\
1,78\end{array}$ & $\begin{array}{c}61,75 \\
30,5 \\
9,58 \\
2,65\end{array}$ & $\begin{array}{r}58,33 \\
38,44 \\
9,79 \\
3,00\end{array}$ & $\begin{array}{r}59,67 \\
52,96 \\
9,39 \\
1,90\end{array}$ & $\begin{array}{l}\mu \mathrm{g} / 100 \mathrm{ml} \\
\mu \mathrm{g} / 100 \mathrm{ml} \\
\mathrm{mg} / 100 \mathrm{ml} \\
\mathrm{mg} / 100 \mathrm{ml}\end{array}$ \\
\hline $\begin{array}{l}\text { Glucose } \\
\text { BOH } \\
\text { Urée } \\
\text { Albumine } \\
\text { Céruloplasmine } \\
\text { AGR } \\
\text { Cholestérol } \\
\text { Phospholipides } \\
\text { Triglycérides }\end{array}$ & $\begin{array}{c}86,9 \\
0,025 \\
21,25 \\
31,88 \\
39,68 \\
0,129 \\
21,99 \\
26,84 \\
21,66\end{array}$ & $\begin{array}{c}54,55 \\
0,002 \\
17,48 \\
34,52 \\
34,8 \\
0,29 \\
18,78 \\
21,11 \\
26,67\end{array}$ & $\begin{array}{c}98,75 \\
0,037 \\
55,57 \\
34,25 \\
35,17 \\
0,145 \\
21,25 \\
24,25 \\
11,79\end{array}$ & $\begin{array}{c}62,33 \\
0,018 \\
46,81 \\
32,8 \\
36 \\
0,204 \\
20,28 \\
22,55 \\
34,39\end{array}$ & $\begin{array}{c}49,8 \\
0,008 \\
42,91 \\
32,05 \\
33,65 \\
0,139 \\
17,77 \\
19,42 \\
25,52\end{array}$ & $\begin{array}{c}\mathrm{mg} / 100 \mathrm{ml} \\
\mathrm{mmol} / \mathrm{l} \\
\mathrm{mg} / 100 \mathrm{ml} \\
\mathrm{g} / \mathrm{l} \\
\mathrm{UO} \\
\mathrm{mmol} / \mathrm{l} \\
\mathrm{mg} / 100 \mathrm{ml} \\
\mathrm{mg} / 100 \mathrm{ml} \\
\mathrm{mg} / 100 \mathrm{ml}\end{array}$ \\
\hline $\begin{array}{l}\text { GGT } \\
\text { GLDH }\end{array}$ & $\begin{array}{l}8,4 \\
2,4\end{array}$ & $\begin{array}{r}8,8 \\
13,4\end{array}$ & $\begin{array}{l}5,7 \\
3,0\end{array}$ & $\begin{array}{r}8,8 \\
27,4\end{array}$ & $\begin{array}{r}8,5 \\
16,4\end{array}$ & $\begin{array}{l}\text { UI } \\
\text { UI }\end{array}$ \\
\hline
\end{tabular}

TABLEAU III Matrice des corrélations entre les différents paramètres plasmatiques mesurés sur le dromadaire à Djibouti. Seuil de signification à 1 p. cent pour les chiffres en caractères gras et 5 p. cent pour les autres.

\begin{tabular}{|c|c|c|c|c|c|c|c|c|c|c|c|c|c|c|c|}
\hline & TRG & $\mathrm{CHO}$ & $\mathrm{PHO}$ & $\mathrm{BOH}$ & GLU & AGR & URE & ALB & CER & CUI & ZIN & CAL & $M G$ & GGT & GLD \\
\hline $\begin{array}{l}\text { Triglycérides } \\
\text { Cholestérol } \\
\text { Phospholipides } \\
\text { BOH } \\
\text { Glucose } \\
\text { AGL } \\
\text { Urée } \\
\text { Albumine } \\
\text { Céruloplasmine } \\
\text { Cuivre } \\
\text { Zinc } \\
\text { Calcium } \\
\text { Magnésium } \\
\text { Gamma GT } \\
\text { GLDH }\end{array}$ & $\begin{array}{r}.275 \\
.296 \\
-.339\end{array}$ & .674 & $\begin{array}{l}.439 \\
.361 \\
.294\end{array}$ & $\begin{array}{r}.362 \\
-.358 \\
-.372\end{array}$ & $\begin{array}{l}.434 \\
.430\end{array}$ & & -.393 & $\begin{array}{r}1 \\
-.295 \\
.291 \\
-.307 \\
- \\
-\end{array}$ & .720 & .394 & -.275 & .313 & & .467 & \\
\hline & TRG & $\mathrm{CHO}$ & $\mathrm{PHO}$ & $\mathrm{BOH}$ & GLU & AGR & URE & ALB' & CER & CUI & ZIN & $\mathrm{CAL}$ & MG & GGT & GLD \\
\hline
\end{tabular}


TABLEAU IV Valeur de F pour les variables significativement discriminantes de la population cameline en fonction de l'âge (deux niveaux : adulte et jeune), du sexe (deux niveaux : mâle ou femelle) et de la région (cinq régions).

\begin{tabular}{|c|c|c|c|c|c|}
\hline \multicolumn{2}{|c|}{ Age } & \multicolumn{2}{|c|}{ Sexe } & \multicolumn{2}{|c|}{ Région } \\
\hline $\begin{array}{l}\text { Paramètres } \\
\text { discriminants }\end{array}$ & $\begin{array}{l}\text { Valeur } \\
\text { de F }\end{array}$ & $\begin{array}{l}\text { Paramètres } \\
\text { discriminants }\end{array}$ & $\begin{array}{l}\text { Valeur } \\
\text { de F }\end{array}$ & $\begin{array}{l}\text { Paramètres } \\
\text { discriminants }\end{array}$ & $\begin{array}{l}\text { Valeur } \\
\text { de F }\end{array}$ \\
\hline $\begin{array}{l}\text { Triglycérides } \\
\text { Zinc } \\
\text { Albumine } \\
\text { Cholestérol } \\
\text { Glucose } \\
\text { Céruloplasmine }\end{array}$ & $\begin{array}{c}21,26^{\star \star *} \\
6,76^{\star} \\
4,78^{\star} \\
4,56^{\star} \\
4,24^{\star} \\
4,18^{\star}\end{array}$ & $\begin{array}{l}\text { Céruloplasmine } \\
\text { Cuivre } \\
\text { Urée } \\
\text { Phospholipides } \\
\text { Magnésium } \\
\text { Glucose }\end{array}$ & $\begin{array}{c}24,19^{\star * *} \\
14,21^{* * *} \\
7,16^{*} \\
6,77^{*} \\
4,19^{\star} \\
4,09^{\star}\end{array}$ & $\begin{array}{l}\text { Magnésium } \\
\text { Urée } \\
\text { Céruloplasmine } \\
\text { BOH } \\
\text { Cuivre } \\
\text { Glucose }\end{array}$ & $\begin{array}{r}17,18^{\star * \star} \\
14,85^{\star \star \star} \\
14,80^{\star * \star} \\
14,61^{* \star *} \\
12,78^{\star \star \star} \\
9,44^{\star \star \star}\end{array}$ \\
\hline \multicolumn{6}{|c|}{ Pourcentage de bien-classés } \\
\hline \multicolumn{2}{|c|}{94,2} & \multicolumn{2}{|c|}{90,4} & \multicolumn{2}{|c|}{98,1} \\
\hline
\end{tabular}

Seuils de signification : *** $P<0,001 ;{ }^{* *} P<0,01 ; * P<0,05$.

Les enzymes hépatiques atteignent des seuils "critiques" (si on se réfère aux normes de l'espèce bovine) dans la région du Sud-Ouest et surtout dans la région d'AliSabieh.

\section{Analyses multidimensionnelles}

\section{Analyse en composantes principales sur les données centrées réduites}

On analyse un tableau ( $\mathrm{x}$ j) où $\mathrm{i}$ représente les individus (52 dromadaires) et j les valeurs plasmatiques des 15 paramètres mesurés. On ne commentera que la matrice des corrélations (tabl. III), les résultats graphiques de l'ACP n'apportant que peu d'informations intéressantes.

Les coefficients de corrélation les plus significatifs sont relevés entre le cuivre et la céruloplasmine $(0,720)$ d'une part, entre phospholipides et cholestérol $(0,674)$ d'autre part. Les enzymes hépatiques sont également bien corrélées entre elles $(0,467)$.

\section{L'analyse discriminante}

Elle est appliquée sur le tableau des données initiales (i $x$ j) en fonction, respectivement, de l'âge, du sexe et de l'origine géographique des animaux (tabl. IV). Les paramètres les plus discriminants sont les triglycérides pour l'âge, le cuivre et la céruloplasmine pour le sexe. Cependant, il apparaît nettement que l'on peut discriminer de façon plus marquée les animaux, en fonction de leur origine géographique. Les paramètres témoins du niveau énergétique (glucose, $\mathrm{BOH}$ ) et azoté (urée) et les paramètres minéraux (magnésium, cuivre) dépendent très fortement de la région d'origine des animaux.
La projection des individus sur le plan défini par les axes discriminants 1 et 2 (fig. 1) permet de visualiser clairement l'opposition du groupe 1 (Rift de l'Afar) au groupe 5 (région du Sud-Ouest) sur l'axe horizontal, et les groupes 3 et 4 (régions d'Ali-Sabieh, du Bara Yer et élevages périurbains) au groupe 2 (plaine côtière d'Obock) sur l'axe

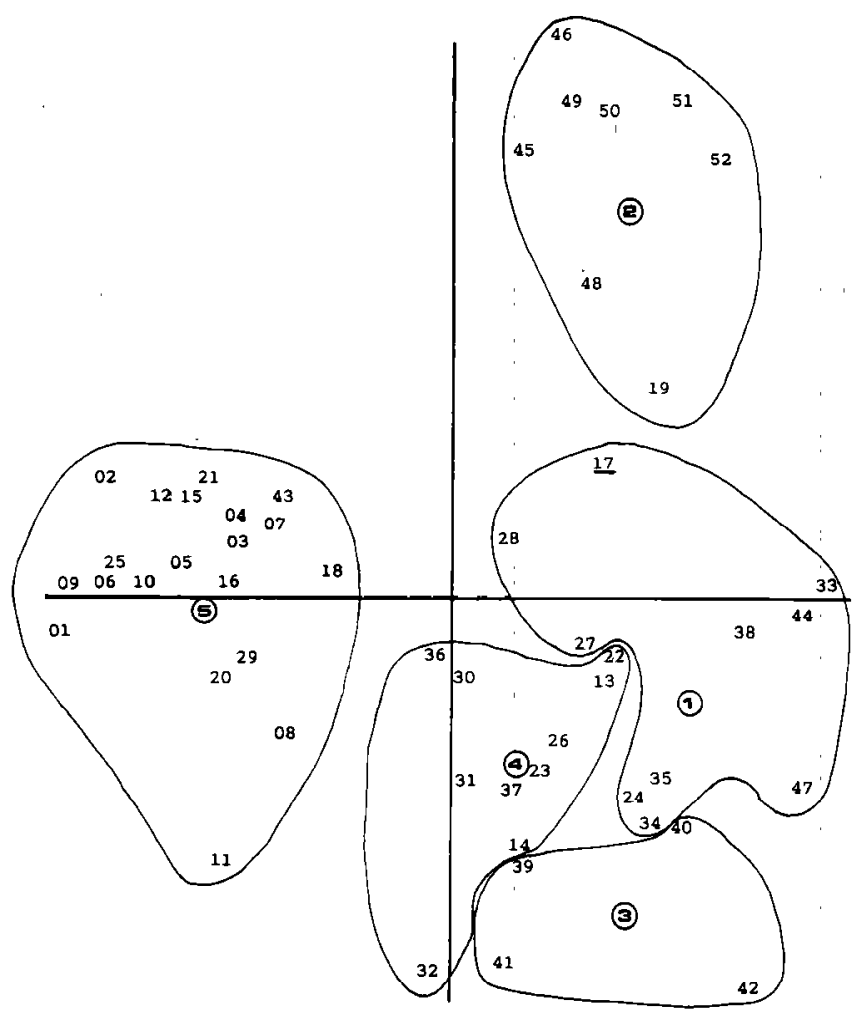

Fig. 1 : Projection des individus (52 dromadaires) et des centres de gravité des cinq régions sur le plan défini par les axes discriminants. 
vertical. Un seul individu est mal classé. II s'agit d'une jeune femelle $\left(n^{\circ} 17\right)$ qui, bien qu'appartenant à la région d'Obock (groupe 2), présente un profil plasmatique plus proche des animaux de la région du Rift Afar (groupe 1).

L'ensemble de ces résultats indique donc l'existence de liens entre les oligo-éléments et le statut énergétique et protéique des animaux, d'une part, et la prééminence du facteur géographique pour discriminer les populations de dromadaires du point de vue de leur profil métabolique d'autre part. La discussion abordera essentiellement ces deux points.

\section{DISCUSSION}

La diversité des sexes, des âges, des stades physiologiques et des ressources alimentaires sur un nombre limité d'animaux constitue sans doute une restriction à la signification des résultats mais permet aux valeurs globalement observées d'être considérées comme représentatives de la diversité des situations.

\section{Valeurs observées chez le dromadaire}

D'après CHANDRASENA et al. (15), le dromadaire présente une glycémie normale de l'ordre de 80 à $140 \mathrm{mg} / 100 \mathrm{ml}$, sensiblement supérieure à celle observée chez les autres ruminants. A l'inverse, les taux de $\mathrm{BOH}$ sont très inférieurs à ceux des autres espèces de ruminants (16). Or, les valeurs observées à Djibouti sont nettement plus faibes pour la glycémie et légèrement supérieures pour le $\mathrm{BOH}$, signifiant sans doute un fort déficit énergétique.

Les teneurs en AGL du dromadaire sont comparables à celles des bovins, contrairement aux taux de cholestérol et de phospholipides, très inférieurs, et au taux de triglycérides, légèrement supérieur (22). Ces résultats corroborent ceux, partiels, d'autres auteurs $(17,32,36,37$, 45).

Les références concernant l'urémie chez le dromadaire sont nombreuses et très variables mais comparables à nos observations $(2,12,37)$. II en est de même pour l'albuminémie $(28,37)$.

La calcémie et la magnésémie apparaissent tout à fait comparables aux valeurs de la littérature pour le dromadaire $(12,37,41,46)$ et comparables à celles des bovins $(3,25)$.

Les valeurs plasmatiques en oligo-éléments (cuivre, zinc), peu nombreuses dans la littérature, oscillent dans la majorité des cas entre 70 et $120 \mu \mathrm{g} / 100 \mathrm{ml}(1,3,21$, $24,43,46)$, ce qui correspond aux valeurs généralement observées chez les bovins, bien que la comparaison inter-espèce dans une même zone géographique indique que les dromadaires présentent en moyenne des cuprémies plus élevées $(22,23,43)$. Les valeurs moyennes relevées à Djibouti paraissent donc plutôt plus faibles (moy. $=60,7 \mu \mathrm{g} / 100 \mathrm{ml}$ ), mis à part les résultats de FAYE et GRILLET (23) dans la vallée de l'Awash, en Éthiopie (moy. $=45 \mu \mathrm{g} / 100 \mathrm{ml}$ ).

Les taux de zinc plasmatique observés à Djibouti sont très inférieurs à ceux relevés par divers auteurs $(21,24$, 31 ). Seuls les résultats d'ABDALLA et al. (1) sont comparables (moy. $=41 \mu \mathrm{g} / 100 \mathrm{ml}$ ).

On dispose de très rares données sur les enzymés hépatiques du dromadaire. ELDIRDIRl et al. (20) observent des taux de GGT légèrement supérieurs aux nôtres $(19,6 \pm 5,0 \mathrm{UI} / \mathrm{l})$ mais proches de ceux d'ABDALLA et al. en $1988(18,0 \pm 7,4 \mathrm{UI} / \mathrm{l})$, alors que ORLIAC (37) relève des valeurs similaires à nos résultats $(11,0 \pm 6 \mathrm{UI} / \mathrm{l})$. Aucune référence n'a été trouvée concernant le taux de GLDH plasmatique dans cette espèce.

Les variations liées à l'âge et au sexe commentées dans la littérature sont éminemment contradictoires et nos résultats ont montré que le facteur de variation le plus important était représenté par la région.

\section{Relations entre les différents paramètres plasmatiques}

La céruloplasmine, enzyme à activité oxydasique associée à 6 ou 8 atomes de cuivre, est donc étroitement corrélée à la cuprémie. Le coefficient de corrélation observé dans notre échantillon $(0,720)$ est hautement significatif, mais l'examen du nuage de points (fig. 2) semble indiquer que pour les valeurs très basses de la cuprémie l'activité oxydasique de la céruloplasmine n'est pas modifiée, ce qui avait déjà été observé en Éthiopie (23). L'équilibre de la ration influe sur la digestibilité des oligo-éléments (33).

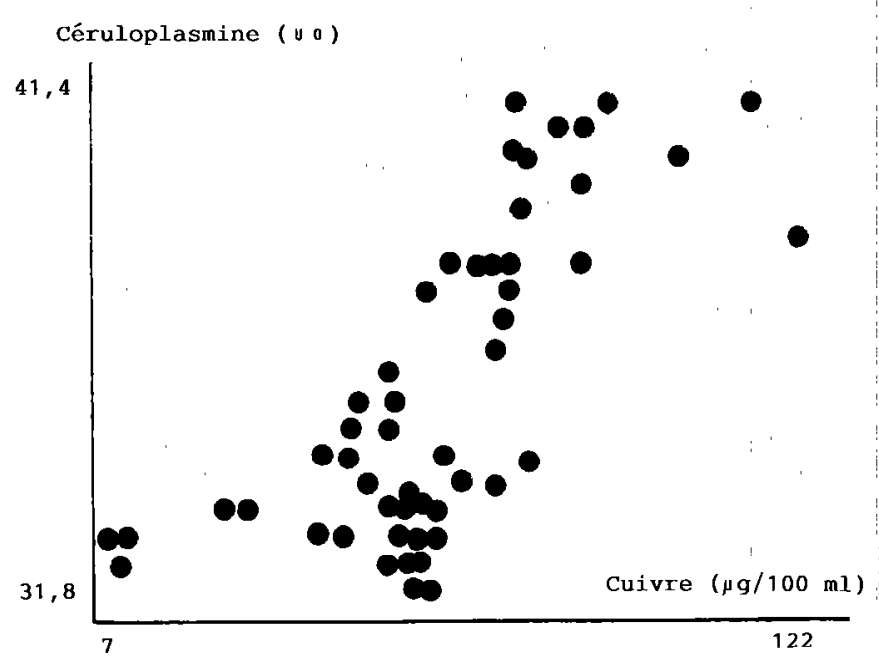

Fig. 2 : Variations du taux de céruloplasmine en fonction de la cuprémie plasmatique chez le dromadaire de Djibouti. 
On note d'ailleurs une relation entre les hypocuprémies et les taux élevés d'AGL et de phospholipides : les animaux en déficit protéo-énergétique (qui ont donc tendance à mobiliser leurs réserves corporelles) présentent une plus faible capacité d'utilisation des oligo-éléments de la ration. Ceci peut se traduire par un statut plasmatique déficitaire.

Phospholipides et cholestérol sont des éléments constitutifs de lipoprotéines et sont associés aux mêmes fonctions au sein de ces macromolécules. En conséquence, il est parfaitement logique d'observer un coefficient de corrélation très significatif. En revanche, les relations lipidesminéraux sont plus complexes. Divers auteurs ont observé des relations entre hypocalcémie, hypomagnésémie et déviation du métabolisme lipidique (lipolyse) chez la vache laitière (36).

Chez les ruminants, la lipomobilisation s'accompagne toujours d'une hypomagnésémie. D'ailleurs, on constate que les animaux de la région d'Obock sont les plus déficients en magnésium. Or, leur glycémie est faible et le taux d'AGL très élevé par rapport aux animaux des autres régions, ce qui semble aller dans le sens d'une lipomobilisation accrue sur des individus en déficit énergétique.

YAGIL (47) observe chez le dromadaire en phase de déshydratation une augmentation de l'urémie et de la magnésémie et une baisse du calcium. On ne possède pas de données concernant l'état éventuel de déshydratation dans le cadre de cette étude.

L'hypercuprémie d'origine inflammatoire s'accompagne d'une diminution des taux plasmatiques d'albumine (35) et le parasitisme intestinal induit des pertes massives en albumine chez le mouton (30). Ceci peut sans doute expliquer en partie les corrélations négatives observées entre albumine et céruloplasmine (protéine d'inflammation) et entre l'albumine et la GGT (enzyme témoin de l'atteinte notamment d'origine parasitaire des canaux biliaires).

La corrélation positive entre la GGT et la GLDH $(0,647)$ est comparable à celle observée chez les bovins $(0,575)$ par BARNOUIN et PACCARD (9), mais ce coefficient dépend essentiellement du niveau des atteintes hépatiques (4).

\section{Variations géographiques}

Bien que par leurs déplacements incessants les dromadaires puissent tempérer l'effet "régional" lié à une homogénéité des ressources fourragères, l'origine géographique des animaux dans cette étude paraît fortement marquer le profil sanguin moyen. On ne discutera, dans cette partie, que des effets remarquables.
Les animaux en provenance du Rift Afar (région 1) présentent un profil moyen laissant supposer une alimentation d'assez bonne qualité, offerte par la dominante ligneuse de la ration, essentiellement composée de feuilles d'Acacia (2) dont on sait la richesse en matières azotées (26).

Dans la région d'Obock, le profil observé signe surtout un état général déficitaire. Sur les troupeaux pâturant dans les formations côtières à Avicennia et Ceriops (mangroves), les déficits minéraux sont particulièrement prononcés (25). Ils sont liés à des taux très faibles en minéraux des formations végétales consommées (22) mais, compte tenu des valeurs également très basses de la glycémie et de l'urémie observées dans cette région, le déficit paraît aussi énergétique et azoté. Cette situation de "carence" a déjà été observée par GODET et al. (29) mais sa nature non précisée.

La combinaison d'une hypomagnésémie relativement marquée et d'une lipomobilisation traduite par une montée des AGL et une glycémie faible a déjà été évoquée. Ceci appuie encore l'hypothèse d'un déficit énergétique grave.

Le niveau énergétique de la ration alimentaire des chamelles en élevage périurbain est largement supérieur à celui des autres dromadaires du pays. Ces animaux reçoivent une ration à base de grains (sorgho-maïs), parfois associés à des fourrages verts. Les apports azotés paraissent excessifs et l'urémie moyenne $(55,5 \mathrm{mg} / 100 \mathrm{ml})$ est bien plus élevée que les normes généralement observées dans cette espèce (6).

Une partie des animaux de la région d'Ali-Sabieh-Bara Yer pâturait dans les fourrés à Salvadora persica, plante buissonnante pauvre en cuivre et zinc, riche en molybdène, soufre, sélénium et magnésium (22) et d'assez bonne valeur azotée (26). Or ces animaux présentent des taux de magnésium sanguin bien supérieurs à ceux relevés par d'autres auteurs (12), ainsi que des urémies très fortes, ce qui pourrait signifier une atteinte rénale. Peuton imputer également à ce profil minéral particulier des ressources alimentaires les taux élevés en GLDH témoins d'une atteinte hépatique non parasitaire ? En effet, la combinaison d'une hypermagnésémie, d'une urémie assez forte et d'une montée de la GLDH pourrait faire soupçonner un processus toxique hépato-rénal dont la consommation exclusive de fourrés à Salvadora et Cadaba pourrait être à l'origine. D'ailleurs, les animaux broutant ce type de végétation présentent des diarrhées nauséabondes qui rappellent des symptômes de molybdénose (22).

La région du Sud-Ouest, plus hétérogène, ne révèle pas de variations remarquables du profil. La montée de GLDH ou le déficit magnésique observé chez quelques animaux de la plaine d'Agna expliquent les moyennes observées sur l'ensemble de la région. Le déficit en cuivre chez les troupeaux de la région du lac Abbé affecte l'ensemble des espèces pâturant dans la zone (25) et est associé au 
B. Faye C. Mulato

statut minéral des fourrages caractérisé par un excès de molybdène et de soufre, éléments antagonistes bien connus du cuivre (34).

\section{CONCLUSION}

La région naturelle d'origine des animaux représente le facteur le plus discriminant des profils métaboliques. Ce résultat permet de considérer qu'en zone tropicale extensive le statut nutritionnel des animaux est associé essentiellement à l'espace utilisé par les troupeaux, et qu'on peut définir ainsi un statut éconutritionnel.

FAYE (B.), MULATO (C.). Factors of variation of mineral, biochemical and enzymatic parameters in the blood plasma of dromedaries in Djibouti. Revue Flev. Méd. vét. Pays trop., 1991, 44 (3) : 325-334

Fifteen blood plasma parameters (mineral, biochemical and enzymatic) in $\mathbf{5 2}$ dromedaries ( 27 adult females, 7 adult males, 5 young males and 13 young females) from five natural areas in Djibouti were analysed. According to observed values, Djiboutian dromedaries exhibited trace element deficiencies concerning copper $(60.7 \mu \mathrm{g} / 100 \mathrm{ml})$ and zinc $(46.2 \mu \mathrm{g} / 100 \mathrm{ml})$ but not calcium $(9.5 \mathrm{mg} / 100 \mathrm{ml})$ or magnesium $(2.3 \mathrm{mg} / 100 \mathrm{ml})$ although the standard deviation was large in the latter case (1.1-3.9). Moreover, dromedaries in Djibouti seemed to be energy deficient $(63.7 \mathrm{~g} / 100$ ml for glucose, $\mathbf{0 . 0 2 5} \mathrm{mmole} / \mathrm{l}$ for BOII, $0.17 \mathrm{mmole} / \mathrm{l}$ for FFA), but the dietary protein level seemed to be satisfactory (urea $35.9 \mathrm{mg} / 100 \mathrm{ml}$, albumine $32.7 \mathrm{~g} / \mathrm{l}$ ). The metabolic peculiarities of dromedaries explain the values of plasma lipids (cholesterol 19.5, phospholipid 22.4 and triglyceride $26.6 \mathrm{mg} / 100 \mathrm{ml}$ ). The geographical factor prevails over the zootechnical factor (age, sex) for explaining the differences between observed profiles. The characteristics between the five natural areas are discussed in relation to mean profile, observed on the animals. Key words : Dromedary Biochemistry - Enzyme - Mineral content - Plasma - Djihouti.
En République de Djibouti, on peut globalement constater que les dromadaires sont plutôt en déficit minéral (cuivre et surtout zinc) et que la couverture énergétique n'est pas assurée pour la plupart des animaux.

\section{REMERCIEMENTS}

Nous tenons à remercier le Dr LAURENT, du Ministère de la Coopération, et le Dr.KAMIL, du Service de l'Élevage et des Pêches de Djibouti, sans qui cette étude n'aurait pas été possible.

Nous remercions également J.P. CHACORNAC, J.C. TRESSOL, Françoise LESCOURRET et G. SAINT-MARTIN pour l'aide qu'ils nous ont apportée.

FAYE (B.), MULATO (C.). Factores de variación de los parámetros proteo-energéticos, enzimaticos y minerales del plasma en el dromádario de Djibouti. Revue Élev. Méd. vét. Pays trop., 1991,44 (3) : 325-334

Se analizaron 15 parámetros bioquímicos a partir del plasma de 52 dromedarios ( 27 hembras adultas, 13 hembras jovenes, 7 machos adultos y 5 machos jovenes) proviniendo de cinco regiones naturales de Djibouti. Los resultados evidencian estados de subcarencia de oligoelementos (cobre : $60,7 \mu \mathrm{g} / 100 \mathrm{ml}$, cinc : $46,2 \mu \mathrm{g} / 100 \mathrm{ml}$ ), de ninguna manera de calcio $(9,5 \mathrm{mg} / 100 \mathrm{ml})$, ni de magnesio $(2,3 \mathrm{mg} / 100 \mathrm{ml})$, aunque en el último caso las diferencias sean importantes (1,1-3,9). Por otro lado, los dromedarios de Djibouti parecen tener un deficit energético $(63,7 \mathrm{mg} / 100 \mathrm{ml}$ para el glucosis, $0,025 \mathrm{mmol} / \mathrm{l}$ para el BOH, $0,17 \mathrm{mmol} / 1$ para los ácidos grasos libres), pero el nivel nitrogenado alimenticio es globalmente satisfactorio (urea $35,9 \mathrm{mg} / 100 \mathrm{ml}$, albumina $32,7 \mathrm{~g} / \mathrm{l}$ ). Los lípidos plasmáticos (colesterol, fosfolípidos, triglicéridos) indican las particularidades metabolicas del dromedario (respectivamente 19,5, 22,4 y 26,6 mg/100 ml). El factor geográfico es preponderante sobre los factores zootécnicos para explicar las diferencias entre los perfiles observados. Se discuten las características de las cinco regiones con relación al perfil medio observado en los animales. Palabras claves : Dromedario - Bioquímica - Enzima - Tasa de elementos minerales - Plasma - Djibouti.

\section{BIBLIOGRAPHIE}

1. ABD $\mathrm{LL}$ L (O.M.), WASFI (I.A.), GADIR (F.A.). The Arabian race camel normal parameters. I. Haemogram, enzymes and minerals. Comp. Biochem. Physiol., 1988, 90 (2) :237-239.

2. ABDO (M.S.), HASSANIEN (N.M.), MANNA (M.E.), HAMED (M.). Electrophoretic pattern of serum proteins in the Arabian camel. Indian vet. J., 1987, 64 (10) : 841-844.

3. ABU DAMIR (H.), TARTOUR (G.), ADAM (E.I.). Short communication : mineral contents in livestock in Eastern Sudan. Trop. Anim. Hlth Prod., 1983, 15 : 15.

4. AISSAOUI (C.). Enzymes circulantes (OCT, GLDH, GGT) à localisation hépatique chez la vache laitière. Facteurs de variation physiologiques, nutritionnels et relation avec la pathologie. Thèse doct., Úniv. Languedoc, Montpellier. $1990.230 \mathrm{p} .\left(\mathrm{n}^{\circ} 91\right.$ ).

5. ALLAIN (C.C.), POON (L.S.), CHAN (C.S.G.), RICHMOND (N.), FU (P.C.). Enzymatic determination of total serum cholesterol. Clin. Chem., 1974, 20 (4) : 470-475. 
6. ATEEQ (G.), KOUIDER (S.), KOLB (E.). Untersuchungen über den Gehalt des Blutes von Kamelen an Erythrozyten, an Hämoglobin und an Leukozyten, über den Anteil der verschiedenen Leucozytenformen in Blut sowie überden Gehalt des Serums an Gesamteiweiss, an Harnstoff und an Cholesterin sowie an AspAT un dan AlaAT in Abhängigkeit vom Alter und vom Geschlecht. Arch. exp. VetMed., 1984, 38 (5) : 664-675.

7. AUDRU (J.), CÉSAR (J.), FORGIARINI (G.), LEBRUN (J.P.). La végétation et les potentialités pastorales de la République de Djibouti. Maisons-Alfort, IEMVT, 1987. 384 p.

8. BARNOUIN (J.), EL IDILBI (N.), CHILLIARD (Y.), CHACORN $\Lambda$ C (J.P.), LEFAIVRE (R.). Micro dosage automatisé sans déprotéinisation du 3-hydroxybutyrate plasmatique chez les bovins. Annls Rech. vet., 1986,17 (2) : 129-139.

9. BARNOUIN (J.), PACCARD (P.). Facteurs de risque nutritionnels de la pathologie hépatique dans les troupeaux bovins laitiers en France. Can. vet.J., 1988, 29 : $915-920$.

10. BEAUX (M.F.), GOUET (H.), GOUET (J.P.), PHILIPPEAU (G.), TRANCHEFORT (J.), VERNEAU (M.). Manuel d'utilisation du logiciel STAT-ITCF. Paris, ITCF, 1987.

11. BELLANGER (J.), LAMAND (M.). Méthode de dosage du cuivre et du zinc plasmatique. Bull. tech. Cent. Rech. Zoot. vét. Theix, $1975,20: 53-54$

12. BIAGI (G.), SALUTINI (E.). Sul comportamento della calcemia, fosforemia e magnesemia nel Camelus dromedarius somalo di diversa eta e sesso. Nota Ill. Annali Fac. Med. vet. Univ. Pisa, 1982, 35 : 201-216.

13. BUCOLO (G.), DAVID (H.). Quantitative determination of serum triglycerides by the use of enzymes. Clin. Chem., 1973, 19: 476482.

14. CHACORNAC (J.P.), BARNOUIN (J.), RABOISSON (T.). Micro-dosage automatisé de la céruloplasmine plasmatique par mesure de l'activité oxydasique chez les bovins et les ovins. Reprod. Nutr. Dév., 1986, 26 (2a) : 417-427.

15. CHANDRASENA (L.G.), EMMANUEL (B.), GILANPOUR (H.). A comparative study of glucose metabolism between the camel and the sheep. Comp. Biochem. Physiol., 1979, 62:837-840.

16. CHANDRASENA (L.G.), EMMANUEL (B.), HAMAR (D.W.), HOWARD (B.R.). A comparative study of ketone body metabolism between the camel and the sheep. Comp. Biochem. Physiol., 1979, 64: 109-112.

17. CHIERICATO (G.M.), WARFA (A.A.), SCHIAPELLI (M.P.). Influenza del sesso su alcune variabili' ematochimiche del dromedario. Riv. Zootec. Vet., 1986, 14 (3) : 196-199.

18. CHILLIARD (Y.), BAUCHART (D.), BARNOUIN (J.). Determination of plasma non-esterified fatty acid in herbivores and man a comparison of values obtained by manual or automatic chromatographic, titrimetric, colorimetric and enzymatic methods. Reprod. Nutr. Dév., 1984, 24 (4) : 469-482

19. DON (S.), MIYADA (V.), BAYSINGER (S.), NOHICA (R.M.), NAKAMURA (N.), Albumine quantification by Dye Binding and salt fractionation technique. Clin. Chem., 1972, 18 (1): 52-56.

20. ELDIRDIRI (N.I.), SULIMAN (H.B.), SHOMMEIN (A.M.). Normal serum activities of some diagnostic enzymes in dromedary camcl in Sudan. Vet. Res. Commun., 1987. 11 (3) : 201-203.

21. ELTOHAMY (M.M.), SALAMA (A.), YOUSEF (A.E.A.). Blood constituents in relation to the reproductive state in she-camel (Camelus dromedarius). Beitr. trop. subtrop. Landwirtsch. TropenVetMed., 1986, 24 (4) : 425-430.

22. FAYE (B.). Statut nutritionnel du bétail dans la République de Djibouti. Rapport de mission. INRA Theix, Paris, Ministère de la Coopération, $1989.112 \mathrm{p}$.

23. FAYE (B.), GRILLET (C.). La carence en cuivre chez les ruminants domestiques de la région d'Awash (Éthiopie). Revue Élev Méd. vét. Pays trop., 1984, 37 (1): 42-60.

24. FAYE (B.), GRILLET (C.), TESSEMA (A.). Teneur en oligo-éléments dans le fourrage et le plasma des ruminants domestiques en Éthiopie. Revue Élev. Méd. vét. Pays trop., 1986, 39 (2) : 227-237.

25. FAYE (B.), KAMIL (B.), LABONNE (M.). Teneur en oligo-éléments dans les fourrages et le plasma des ruminants domestiques en République de Djibouti. Revue Élev. Méd. vét. Pays trop., 1990, 43 (3) : 365-373.

26. FAYE (B.), TISSERAND (J.L.). Problèmes de la détermination de la valeur alimentaire des fourrages prélevés par le dromadaire Options méd., Sér. Séminaires, 1989, 2 : 61-65.

27. FENELON (J.P.). Qu'est-ce que l'analyse de données? Paris, Lefonen, 1981

28. GHOSAL (A.K.), APPANNA (T.C.), DWARAKNATH (P.K.). A note on the effect of short-term water deprivation on certain blood characteristics in the camel. Indian J. Anim. Sci., 1975, 45 (2) : 105-108.

29. GODET (J.), GUEDDA (M.), SAID-ELMI, ABBAS DOGALI. Notes sur l'élevage camelin en République de Dijibouti. ISERT rapport section Sciences Humaines. 1985. Pathologie et Alimentation. Djibouti. P. 32-41.

30. GRILLET (C.), FAYE (B.). Fractions électrophorétiques des protéines plasmatiques chez la brebis Adale (Éthiopie). Revue Élev Méd. vét. Pays trop., 1987, 40 (1) : 83-88. 


\section{B. Faye C. Mulato}

31. IBRAHIM (M.S.), MOHAMED (A.R.), EL-BALKEMY (F.A.), OMRAN (H.), ALMAKAWI (M.F.). Étude de l'activité de l'Ivomec chez le chameau. Action antiparasitaire et incidence sur l'état général. Res. Bull. Univ. Zagazig, Egypt, 1982.14 p.

32. KHAN (A.A.), KHOLI (I.S.). A note on variations in blood serum cholesterol in camel before and during rut. Indian J. Anim. Sci., $1973, \mathbf{4 3}(2): 1094-1095$.

33. LAMAND (M.). Influence of protein intake on per os zinc deficiency treatment in sheep. Annls Rech. vét., 1985,16 (3) : $285-287$.

34. LAMAND (M.). Influence of molybdene and sulfur on copper metabolism in sheep. Comparison of elemental sulfur and sulfate. Annls Rech. vét., 1989, 20 : 103-106.

35. LAMAND (M.), LEVIEUX (D.). Effects of infection on plasma levels of copper and zinc in ewes. Annls Rech. vét., 1981, 12 (2) : 133136.

36. MAZUR (A.). Contribution à l'étude de l'infiltration lipidique du foie chez la vache laitière en début de lactation. Thèse doct., Univ. Clermont-II, $1986\left(n^{\circ} 40\right)$.

37. ORLIAC (D.). Contribution à l'étude de la biochimie sanguine de dromadaires et de chèvres sahariens. Thèse doct. Vét., Toulouse, $1980\left(\mathrm{n}^{\circ} 71\right)$.

38. PERK (K.), LOBL (K.). A study of the serum proteins and lipoproteins of the camel and their relations to its resistance to heat and thirst. Refuah vet., 1961, 18 (3) : 163-168.

39. RICHARD (D.), GÉRARD (D.). La production laitière des dromadaires Dankali (Éthiópie). Revue Élev. Méd. vét. Pays trop., 1989, 42 (1) : 97-103.

40. ROWLANDS (G.J.). A review of variations in the concentrations of metabolites in the blood of beef and dairy cattle associated with physiology, nutrition and disease, with particular reference to the interpretation of metabolic profiles. Wrld Rev. Nutr. Diet, 1980 , 35: 172-235.

41. SOLIMAN (M.K.), SHAKER (M.). Cytological and biochemical studies on the blood of adult she camels. Indian vet. J., 1967, 44 989-995.

42. TAKAYAMA (M.), ITOH (S.), NAGASAKI (T.), TANIMIZU (I.). A new enzymatic method for determination of serum choline containing phospholipids. Clin. Chem. Acta, 1977, $79: 83-98$.

43. TARTOUR (G.). Copper status in livestock, pasture and soil in Western Sudan. Trop. Anim. Hlth Prod., 1975,7 : 87-94.

44. TOPPS (J.H.), THOMPSON (J.K.). Blood characteristics and the nutrition of ruminants. London, Ministry of Agriculture, Fish and Food, Dept. of Agriculture and Fish for Scotland HMSO, 1984. 34 p.

45. WASFI (I.A.), HAFEZ (A.M.), EL TAYEB (F.M.), EL TAHER (A.Y.). Thyroid hormones, cholesterol and triglyceride levels in the camel. Res. vet. Sci., 1987, $42: 418$.

46. WHABI (A.A.), ABDEL GARDIR (S.E.), NEIMAT (A.A.), IDRIS (O.F.). Plasma electrolytes and minerais of normal camels in the Sudan. In : Camcls and camclids. Uppsala, Cockrill W.R., 1979. P. 431-437.

47. YAGIL (R.). The desert camel. Comparative physiological adaptation. Basel, Karger, 1985. $164 \mathrm{p}$. 